\title{
DNGK: Mit dem COVID-Cube die richtige Entscheidung treffen
}

\section{Um Menschen die Entscheidung für oder gegen eine COVID-}

19-Impfung zu erleichtern, hat das Deutsche Netzwerk

für Gesundheitskompetenz (DNGK) den COVID-Cube

herausgebracht - eine interaktive Online-Entscheidungshilfe

in Würfelform, die informiert und Präferenzen ermittelt.

Mit dem Cube leistet das Netzwerk einen

Beitrag zu guter Impfkommunikation.

\section{Philipp Ollenschläger}

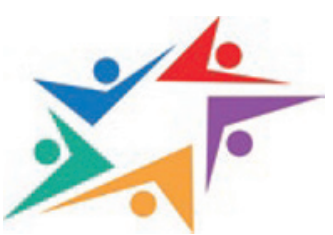

oll ich mich gegen COVID-19 impfen lassen?" Diese Frage treibt derzeit viele Menschen um. Das DNGK bietet Unentschlossenen nun mithilfe des neuen COVID-Cubes kostenfrei die Möglichkeit, leichter eine Antwort auf diese Frage zu finden. Der virtuelle Würfel funktioniert ähnlich wie der Wahl-O-Mat - er beantwortet nicht nur die wichtigsten Fragen zur COVID-Impfung, sondern ermöglicht auch, die Antworten zu bewerten. Jede Ecke des Würfels steht für eine Frage, die für eine Impfentscheidung wichtig sein kann. Wenn man eine Ecke - je nach Endgerät - anklickt oder antippt, erhält man entsprechende Informationen, zudem kann man angeben, ob die Informationen eher für oder eher gegen eine Impfung sprechen.

Der Medizinjournalist Christian Weymayr ist Vorstandsmitglied des DNGK. Er hat den Würfel zusammen mit seinem Partner Christian B. Egbers entwickelt; ursprünglich als Mammographie-Entscheidungshilfe. Aufgrund der Impf-Verunsicherung vieler haben die beiden den Cube nun neu aufgelegt. Im Vergleich zu anderen Angeboten sieht Weymayr bei dem des DNGK einen entscheidenden Vorteil. „Indem man die Ecken des Würfels unabhängig von einer vorgegebenen Reihenfolge abarbeiten kann, hat der Nutzer selbst die Hoheit über die für inn wichtigen Aspekte“, erklärt Weymayr. Häufig geben Entscheidungshilfen vor, was als Nutzen und was als Schaden zu bewerten ist. Dies ist beim COVID-Cube nicht der Fall. - Dank der Würfelform sind die Aspekte nicht linear angeordnet, es gibt also weder ein „wichtiger" und „weniger wichtig“, noch eine Zuordnung in „Vorteile“ und „Nachteile“. Nutzer können jeden Aspekt in sieben Abstufungen von „spricht deutlich dafür" bis "spricht deutlich dagegen" bewerten. Die Angaben werden anonym erfasst und können ausgewertet werden. Das lässt wiederum Rückschlüsse auf den Prozess der Entscheidungsfindung und auf die Präferenzen des Einzelnen zu. Der Würfel birgt somit auch forscherisches Potenzial: Er kann aufzeigen, wie Menschen Entscheidungen treffen.

Mit dem neuen Angebot ermöglicht das DNGK einen niedrigschwelligen Zugang zu einer guten und verlässlichen Entscheidungshilfe. „Der COVID-Cube richtet sich an jeden, - die Texte sind leicht verständ-
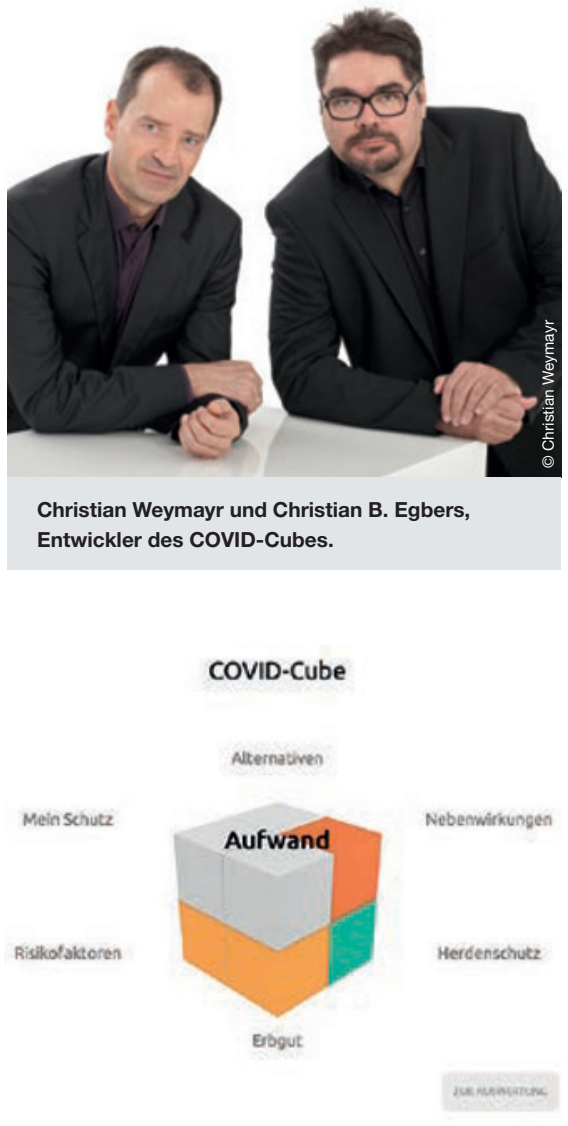

lich, die Handhabung selbsterklärend“, sagt Weymayr. Darüber hinaus hat der Nutzer ein spielerisches, nahezu haptisches Erlebnis. Künftig sind Entscheidungswürfel für weitere medizinische Fragestellungen denkbar, doch nicht nur dafür: Für jede Entscheidung mit mehr als einem Aspekt ist der Cube geeignet. - Er gliedert jede Entscheidung in acht Teilaspekte, liefert gezielt Informationen und verschafft Ratsuchenden so mehr Klarheit.

\section{Weitere Infos:}

https://dngk.de/covid-cube/

https://www.decisioncube.de/

https://christian-weymayr.de/ 